\title{
Effect of Planting Date, Cultivar, and Stage of Plant Development on Incidence of Fusarium Wilt of Lettuce in Desert Production Fields
}

\author{
Michael E. Matheron, The University of Arizona, Yuma Agricultural Center, 6425 West Eighth Street, Yuma 85364; \\ James D. McCreight, U.S. Department of Agriculture, Agricultural Research Service, U.S. Agricultural Research \\ Station, 1636 East Alisal Street, Salinas, CA; and Barry R. Tickes and Martin Porchas, The University of Arizona, \\ Yuma Agricultural Center, 6425 West Eighth Street, Yuma 85364
}

\begin{abstract}
Matheron, M. E., McCreight, J. D., Tickes, B. R., and Porchas, M. 2005. Effect of planting date, cultivar, and stage of plant development on incidence of Fusarium wilt of lettuce in desert production fields. Plant Dis. 89:565-570.

Fusarium wilt of lettuce, first recognized in Japan in 1955, has since been discovered in the United States (California in 1990, Arizona in 2001), Iran (1995), Taiwan (1998), and Italy (2001). In Arizona, the causal agent, Fusarium oxysporum f. sp. lactucae, has been recovered from lettuce plants in 27 different lettuce fields during the 2001 to 2003 production seasons. Studies were initiated to examine the impact of planting date, cultivar, and stage of plant development on the incidence of disease in the field. In 2002 and 2003, tested lettuce cultivars were sown in at least one of the following planting windows; early-season (September), mid-season (October), and late-season (December). Within each planting window, significant differences in disease incidence among lettuce cultivars were noted at plant maturity. The mean incidence of Fusarium wilt on cultivars sown in September, October, and December was 92.3, 15.1, and $2.0 \%$, respectively, in 2002 and 74.2, 5.1, and $0.7 \%$, respectively, in 2003. The mean soil temperatures at the $10-\mathrm{cm}$ depth during the September, October, and December plantings for both years were 26,14 , and $14^{\circ} \mathrm{C}$, respectively. Initial symptoms of Fusarium wilt were apparent as early as 14 days after seeding, with increasing incidence of disease noted as the crop developed and reached maturity. Among all lettuce cultivars planted in September, only one and two cultivars of romaine in 2002 and 2003, respectively, reached maturity with $\leq 5 \%$ incidence of Fusarium wilt, whereas the lowest incidence of disease among crisphead, green leaf, red leaf, or butterhead cultivars was 73.7, 27.0, 20.2, and 65.7\%, respectively, in 2002 and 62.1, 29.0, 100, and $100 \%$, respectively, in 2003 . For October plantings, all romaine cultivars had $\leq 5 \%$ incidence of Fusarium wilt at maturity, whereas disease incidence among tested cultivars of crisphead lettuce in 2002 and 2003 ranged from 0.8 to $66.8 \%$ and 0.3 to $43.3 \%$, respectively. When planted in December, 82 and $88 \%$ of tested cultivars, including all romaine entries, reached maturity with $\leq 1 \%$ incidence of Fusarium wilt. Selection of appropriate lettuce cultivars and planting times should allow successful production of lettuce in the southwestern Arizona production region with minimal or no incidence of disease in fields infested with $F$. oxysporum f. sp. lactucae. On the other hand, successful production of lettuce in infested fields when temperatures favor disease development will not be possible until lettuce cultivars are developed that possess high tolerance or resistance to the pathogen.
\end{abstract}

Fusarium oxysporum f. sp. lactucae was first identified as the cause of a root rot on lettuce (Lactuca sativa L.) in 1955 in Japan (13). Since that initial discovery, three races of the pathogen have been identified on lettuce in that country $(4,5)$. A Fusarium wilt on lettuce was reported in California in 1990, and the causal pathogen was named Fusarium oxysporum f. sp. lactucum (10). Recently, Japanese race 1 and the California pathogen were determined to belong to the same vegetative compatibility group and

Corresponding author: M. E. Matheron

E-mail: matheron@ag.arizona.edu

Accepted for publication 11 January 2005.

DOI: 10.1094/PD-89-0565

(C) 2005 The American Phytopathological Society were considered to be the same forma specialis (4). Lettuce showing symptoms of wilt, yellowing of leaves, and a redbrown to black necrosis of internal taproot and crown tissue was first recognized in 2001 in six different lettuce fields in Arizona. Infected plants were stunted and often died, resulting in significant losses to the grower. $F$. oxysporum was consistently isolated from necrotic taproot tissue from affected field plants, caused symptoms of wilt, yellowing, and root necrosis when lettuce seedlings were inoculated with the fungus, and was of the same vegetative compatibility group and race as an isolate of $F$. oxysporum $\mathrm{f}$. sp. lactucae from lettuce in California reported in $1993(12,14)$. Since the initial discovery in six Arizona lettuce fields in 2001, the pathogen has been recovered from 21 additional fields during the 2002 and 2003 production seasons. The in- fested fields ranged in size from 4 to 16 ha. In addition to Japan and the United States, the pathogen has been reported on lettuce in Iran in 1995 (15), Taiwan in 1998 (9), and Italy in 2001 (8).

Lettuce has become the dominant vegetable crop in southwestern Arizona (Yuma County), with over 28,000 ha devoted to its production. Crisphead (iceberg) lettuce accounts for 20,000 ha, followed by 5,000 ha of romaine and 3,000 ha of green leaf, red leaf, and butterhead. Lettuce seed is sown from early September though December to provide a daily supply of lettuce from November through late March. There are significant differences in soil and air temperatures as well as time required to achieve maturity within this September through December planting season. Lettuce cultivars have been developed for optimum performance within a specific window or time period during the planting season. Some cultivars are bred to endure the high to moderate temperatures (daily maximum ranges from ca. 30 to $45^{\circ} \mathrm{C}$ ) encountered in September and October, others grow best in the moderate to mild temperatures (daily maximum ranges from ca. 25 to $30^{\circ} \mathrm{C}$ ) common from late October through early December, while some cultivars are best suited for the cool to mild temperatures (daily maximum ranges from ca. 10 to $30^{\circ} \mathrm{C}$ ) from December through March.

Lettuce plants displaying symptoms of Fusarium wilt to date have all been found in commercial fields from mid-October through early January. Planting dates for these fields ranged from September to mid-October. Furthermore, 25 of 27 fields in which Fusarium wilt was found were planted to a crisphead cultivar. These observations suggest that lettuce plantings established in September to midOctober, especially plantings of crisphead lettuce, may be more at risk to Fusarium wilt than lettuce plantings made later in the season.

The objective of our research was to examine the impact of planting date, cultivar, and stage of plant development on the resultant incidence of Fusarium wilt of lettuce in the field, with the goal of acquiring data that may facilitate development of disease management guidelines that would enable growers to minimize losses due to Fusarium wilt of lettuce. 


\section{MATERIALS AND METHODS}

Trials were established in a 16-ha commercial field in southwestern Arizona. When planted to crisphead lettuce in the fall of 2001, this field sustained severe crop loss due to Fusarium wilt. Laserleveling and other cultivation practices helped spread the infested soil to produce a uniformly infested field for these studies. In 2002 and again in 2003, each lettuce cultivar was sown in at least one of the following planting windows: early-season (September), mid-season (October), and late-season (December). In 2002, the September, October, and December plantings were located in the western, middle, and eastern one-third of the 16-ha field, respectively. In 2003, all three plantings were made in the portion of the field used for the September planting in 2002, with the September, October, and December plantings located in the western, middle, and eastern one-third of this 5.3-ha area of land. The majority of the lettuce cultivars within each planting date were those that would be planted in the desert southwest production region at that time. The remaining cultivars were included for comparison of disease incidence on the same cultivar among different planting dates or for evaluation of plant material not grown commercially in the desert. Plots were planted and managed using current commercial practices, utilizing raised beds with $92 \mathrm{~cm}$ between bed centers, with each bed containing two rows of lettuce spaced $30 \mathrm{~cm}$ apart. In each planting, the four replicate plots for each tested cultivar were arranged in a randomized complete block design. Each plot contained 600 plants in each of the three plantings in 2002, 180 plants in September 2003, and 550 plants in October and December 2003.

Disease incidence was assessed three times for each planting. Each plant within a plot was counted as diseased if the plant was dead or stunted and displayed the typical wilting and yellowing associated with Fusarium wilt of lettuce. All living plants with these symptoms of Fusarium wilt were not marketable at plant maturity. During the third and final rating, root cortex tissue was examined for the presence of discoloration and necrosis as well. Samples of necrotic root cortex tissue from each lettuce planting were placed on acidified potato dextrose agar to verify the presence of $F$. oxysporum f. sp. lactucae. Soil temperature in the bed was recorded hourly at a depth of $10 \mathrm{~cm}$. Planting dates were 7 September, 17 October, and 6 December in 2002 and 3 September, 21 October, and 18 December in 2003. For 2002 plantings, disease incidence was recorded 2 and 14 October and 8 November 2002 for the 7 September planting; 24 November, 15 December 2002, and 11 January 2003 for the 17 October planting; and 25 January, 15 February, and 22 March 2003 for the 6 December planting. For 2003
Table 1. Incidence of Fusarium wilt on lettuce cultivars at crop maturity after sowing seed at three different time periods for 2 years in a field naturally infested with Fusarium oxysporum f. sp. lactucae

\begin{tabular}{|c|c|c|c|c|c|c|}
\hline \multirow{3}{*}{$\begin{array}{l}\text { Lettuce } \\
\text { cultivar }\end{array}$} & \multicolumn{6}{|c|}{ Percentage of plants dead or diseased $^{\mathrm{z}}$} \\
\hline & \multicolumn{3}{|c|}{ Planting date: 2002} & \multicolumn{3}{|c|}{ Planting date: 2003} \\
\hline & $7 \mathrm{Sep}$ & 17 Oct & 6 Dec & 3 Sep & 21 Oct & 18 Dec \\
\hline \multicolumn{7}{|l|}{ Crisphead } \\
\hline Acacia & $100.0 \mathrm{a}$ & $\ldots$ & $\ldots$ & & $\ldots$ & $\ldots$ \\
\hline Annie & $99.5 \mathrm{ab}$ & & $\ldots$ & $98.6 \mathrm{ab}$ & & \\
\hline AZ 90 & $\ldots$ & $66.8 \mathrm{~cd}$ & $\ldots$ & $\ldots$ & $1.4 \mathrm{jk}$ & $0.8 \mathrm{~cd}$ \\
\hline AZ 2000 & $\ldots$ & $\ldots$ & $10.1 \mathrm{~b}$ & 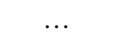 & $\ldots$ & $\ldots$ \\
\hline Beacon & $100.0 \mathrm{a}$ & $72.0 \mathrm{bc}$ & $\ldots$ & $100.0 \mathrm{a}$ & $23.3 \mathrm{~d}$ & $1.6 \mathrm{bc}$ \\
\hline Big Sur & $\ldots$ & $\ldots$ & $0 \mathrm{f}$ & $\ldots$ & $\ldots$ & $0.2 \hbar$ \\
\hline Bubba & & $45.2 \mathrm{i}-\mathrm{k}$ & 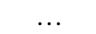 & & $24.7 \mathrm{~d}$ & $2.2 \mathrm{~b}$ \\
\hline Buccaneer & $87 \mathrm{f}-\mathrm{h}$ & $1.2 \mathrm{v}$ & $0 \mathrm{f}$ & $51.0 \mathrm{i}-\mathrm{k}$ & $0.6 \mathrm{k}$ & $0 \mathrm{~d}$ \\
\hline Cavalier & $100.0 \mathrm{a}$ & $\ldots$ & $\ldots$ & $96.7 \mathrm{ab}$ & & $\ldots$ \\
\hline Cibola & $\ldots$ & $62.2 \mathrm{c}-\mathrm{f}$ & $\ldots$ & $\ldots$ & $16.2 \mathrm{e}$ & $\ldots$ \\
\hline Climax & $\ldots$ & $\ldots$ & $0.1 \mathrm{f}$ & $\ldots$ & $\ldots$ & $\ldots$ \\
\hline Cochise 47 & $\ldots$ & $59.5 \mathrm{~d}-\mathrm{g}$ & $\ldots$ & $\ldots$ & $0.3 \mathrm{k}$ & $\ldots$ \\
\hline Colossus & $\ldots$ & $16.2 \mathrm{q}-\mathrm{s}$ & $\ldots$ & $\ldots$ & $\ldots$ & $\ldots$ \\
\hline Coolgreen & $\ldots$ & $\ldots$ & $4.6 \mathrm{c}-\mathrm{e}$ & $\ldots$ & $\ldots$ & $\ldots$ \\
\hline Coolguard & $\ldots$ & $41.0 \mathrm{k}-\mathrm{n}$ & $\ldots$ & $\ldots$ & $3.3 \mathrm{~g}-\mathrm{k}$ & 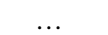 \\
\hline Coyote & . & $14.0 \mathrm{q}-\mathrm{u}$ & 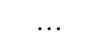 & $99.3 \mathrm{a}$ & $3.3 \mathrm{~g}-\mathrm{k}$ & $0.7 \mathrm{~cd}$ \\
\hline Crusader & 95.2 a-e & $\ldots$ & $0.2 \mathrm{f}$ & $98.6 \mathrm{ab}$ & $\ldots$ & $0.2 \mathrm{~d}$ \\
\hline Daneberg 66 & $\ldots$ & $\ldots$ & $0.2 \mathrm{f}$ & $\ldots$ & $\ldots$ & $0.6 \mathrm{~d}$ \\
\hline Del Oro & $\ldots$ & $44.0 \mathrm{i}-1$ & $\ldots$ & $\ldots$ & $8.5 \mathrm{f}$ & $0 \mathrm{~d}$ \\
\hline Del Rio & 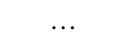 & $6.0 \mathrm{r}-\mathrm{v}$ & $0 \mathrm{f}$ & $100.0 \mathrm{a}$ & & $\ldots$ \\
\hline Desert Heat & $100.0 \mathrm{a}$ & $46.5 \mathrm{i}-\mathrm{k}$ & $10.9 \mathrm{~b}$ & $100.0 \mathrm{a}$ & $0.7 \mathrm{k}$ & $0.4 \mathrm{~d}$ \\
\hline Desert Queen & $100.0 \mathrm{a}$ & $\ldots$ & & $92.5 \mathrm{ab}$ & $\ldots$ & $x^{2}$ \\
\hline Desert Spring & & $\ldots$ & $3.3 \mathrm{~d}-\mathrm{f}$ & & $\ldots$ & $0.6 \mathrm{~d}$ \\
\hline Desert Storm & $100.0 \mathrm{a}$ & $\ldots$ & $\ldots$ & $91.2 \mathrm{ab}$ & $\ldots$ & $\ldots$ \\
\hline Diamond & $\ldots$ & $\ldots$ & $0 \mathrm{f}$ & 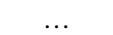 & $\ldots$ & $\ldots$ \\
\hline Diamond Back & 95.2 a-e & $\ldots$ & $0 \mathrm{f}$ & $73.5 \mathrm{de}$ & $\ldots$ & $0 \mathrm{~d}$ \\
\hline Domingos 67 & $\ldots$ & $\ldots$ & $0.1 \mathrm{f}$ & $\ldots$ & $\ldots$ & $0.2 \mathrm{~d}$ \\
\hline Durango & & $\ldots$ & $0 \mathrm{f}$ & $\ldots$ & $\ldots$ & $\ldots$ \\
\hline Emperor & $100.0 \mathrm{a}$ & $\ldots$ & $\ldots$ & & $\ldots$ & $\ldots$ \\
\hline Fallgreen & $100.0 \mathrm{a}$ & $\ldots$ & $\ldots$ & $95.1 \mathrm{ab}$ & $\ldots$ & $\ldots$ \\
\hline Fiorette & & $\ldots$ & $0 \mathrm{f}$ & 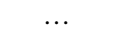 & $\ldots$ & $\ldots$ \\
\hline Fortuna & $100.0 \mathrm{a}$ & $\ldots$ & $\ldots$ & $99.0 \mathrm{ab}$ & $\ldots$ & $\ldots$ \\
\hline Gabilan 1315 & & $\ldots$ & $17.8 \mathrm{a}$ & & $\ldots$ & $0.9 \mathrm{~cd}$ \\
\hline Grand Max & $98.5 \mathrm{a}-\mathrm{c}$ & $\ldots$ & $\ldots$ & $95.9 \mathrm{ab}$ & $\ldots$ & $\ldots$ \\
\hline Green Lightning & & $\ldots$ & $0.2 \mathrm{f}$ & & $\ldots$ & $0.2 \mathrm{~d}$ \\
\hline Green Pack & $87.2 \mathrm{f}-\mathrm{h}$ & $3.0 \mathrm{uv}$ & $\ldots$ & 68.4 ef & & $\ldots$ \\
\hline Grizzly & $\ldots$ & $30.0 \mathrm{n}-\mathrm{p}$ & $\ldots$ & $\ldots$ & $2.2 \mathrm{i}-\mathrm{k}$ & $\ldots$ \\
\hline Headmaster & $\ldots$ & $\ldots$ & $0 \mathrm{f}$ & $\ldots$ & $\ldots$ & $0.2 \mathrm{~d}$ \\
\hline Heatmaster & $100.0 \mathrm{a}$ & $\ldots$ & $\ldots$ & $\ldots$ & $\ldots$ & $\ldots$ \\
\hline Heavy Weight & $\ldots$ & $\ldots$ & $\ldots$ & $98.7 \mathrm{ab}$ & $13.7 \mathrm{e}$ & $\ldots$ \\
\hline Honcho II & - & $46.8 \mathrm{~h}-\mathrm{k}$ & $\ldots$ & $\ldots$ & $25.4 \mathrm{~d}$ & $\ldots$ \\
\hline Husky & $100.0 \mathrm{a}$ & $16.8 \mathrm{q}-\mathrm{r}$ & $0.5 \mathrm{f}$ & $\ldots$ & $\ldots$ & $\ldots$ \\
\hline Icon & $\ldots$ & $\ldots$ & $0.4 \mathrm{f}$ & $\ldots$ & $\ldots$ & $0.3 \mathrm{~d}$ \\
\hline Jackel & & $\ldots$ & $0.2 \mathrm{f}$ & $\ldots$ & $\ldots$ & $0.3 \mathrm{~d}$ \\
\hline Javelina & $97.7 \mathrm{a}-\mathrm{c}$ & $\ldots$ & $\ldots$ & $58.0 \mathrm{~g}-\mathrm{i}$ & $\ldots$ & $\ldots$ \\
\hline Kahuna & $99.5 \mathrm{ab}$ & $\ldots$ & $\ldots$ & $100.0 \mathrm{a}$ & $\ldots$ & $\ldots$ \\
\hline Kofa & $\ldots$ & $40.0 \mathrm{k}-\mathrm{o}$ & $\ldots$ & $\ldots$ & $15.5 \mathrm{e}$ & $\ldots$ \\
\hline Laguna Fresca & $100.0 \mathrm{a}-\mathrm{d}$ & $\ldots$ & $\ldots$ & $99.4 \mathrm{a}$ & $\ldots$ & $0.9 \mathrm{~cd}$ \\
\hline La Quinta & 81.7 h & $\ldots$ & $\ldots$ & & & $\ldots$ \\
\hline Lighthouse & $100.0 \mathrm{a}$ & $66.0 \mathrm{c}-\mathrm{e}$ & $\ldots$ & $100.0 \mathrm{a}$ & $43.3 \mathrm{a}$ & $0.9 \mathrm{a}$ \\
\hline Mighty Joe & & $\ldots$ & $\ldots$ & $100.0 \mathrm{a}$ & $3.9 \mathrm{f}-\mathrm{k}$ & $\ldots$ \\
\hline Milestone & $100.0 \mathrm{a}$ & $\ldots$ & $\ldots$ & $\ldots$ & $\ldots$ & $\ldots$ \\
\hline Mohawk & $100.0 \mathrm{a}$ & $\ldots$ & $\ldots$ & $79.6 \mathrm{~d}$ & $\ldots$ & $\ldots$ \\
\hline Monarch & $100.0 \mathrm{a}$ & $\ldots$ & $\ldots$ & $\ldots$ & $\ldots$ & $\ldots$ \\
\hline Monolith & $\ldots$ & 28.2 op & $\ldots-$ & $95.3 \mathrm{ab}$ & $2.5 \mathrm{i}-\mathrm{k}$ & $0.8 \mathrm{~cd}$ \\
\hline Palma & $100.0 \mathrm{a}$ & $\ldots$ & $\ldots$ & $\ldots$ & $\ldots$ & $\ldots$ \\
\hline Patriot & & $\ldots$ & $0.5 \mathrm{f}$ & & $\ldots$ & $\ldots$ \\
\hline Raider & $92.5 \mathrm{a}-\mathrm{f}$ & & $\ldots$ & $94.1 \mathrm{ab}$ & & $\ldots$ \\
\hline RC 74 & $\ldots$ & $66.8 \mathrm{~cd}$ & $\ldots$ & $\ldots$ & $17.5 \mathrm{e}$ & $\ldots$ \\
\hline Rivergreen & $\cdots$ & $\ldots$ & $0 \mathrm{f}$ & $\ldots$ & $\begin{array}{c}\ldots \\
\text { (continued }\end{array}$ & $\begin{array}{c}\ldots \\
\text { lext page) }\end{array}$ \\
\hline
\end{tabular}

${ }^{\mathrm{z}}$ Each of the four replicate plots for each tested lettuce cultivar, arranged in a randomized complete block experimental design within each planting date, contained 600 plants in all three plantings in 2002, 180 plants in September 2003, and 550 plants in October and December 2003 plantings. Final disease incidence at crop maturity for lettuce planted 7 Sep, 17 Oct, and 6 Dec 2002 was determined 8 Nov 2002, 11 Jan and 22 Mar 2003, respectively; and for plantings sown 3 Sep, 21 Oct, and 18 Dec 2003 was determined on 6 Nov 2003, 26 Jan and 22 Mar 2004, respectively. Entries denoted by “...” were not planted on a given date. Values in each column followed by a different letter are significantly different according to the Duncan multiple range test $(P=0.05)$. 
Table 1. (continued from previous page)

\begin{tabular}{|c|c|c|c|c|c|c|}
\hline \multirow{3}{*}{$\begin{array}{l}\text { Lettuce } \\
\text { cultivar }\end{array}$} & \multicolumn{6}{|c|}{ Percentage of plants dead or diseased ${ }^{z}$} \\
\hline & \multicolumn{3}{|c|}{ Planting date: 2002} & \multicolumn{3}{|c|}{ Planting date: 2003} \\
\hline & $7 \mathrm{Sep}$ & 17 Oct & 6 Dec & 3 Sep & 21 Oct & 18 Dec \\
\hline Sahara & $100.0 \mathrm{a}$ & & $\ldots$ & $100.0 \mathrm{a}$ & $\ldots$ & $\ldots$ \\
\hline Salinas 88 & $\ldots$ & $0.8 \mathrm{v}$ & $\ldots$ & $\ldots$ & $\ldots$ & $\ldots$ \\
\hline Sharpshooter & $73.7 \mathrm{i}$ & $2.1 \mathrm{uv}$ & $0 \mathrm{f}$ & $62.1 \mathrm{f}-\mathrm{h}$ & $1.0 \mathrm{k}$ & $0 \mathrm{~d}$ \\
\hline Silverado & $\ldots$ & $\ldots$ & $0 \mathrm{f}$ & $\ldots$ & $\ldots$ & $\ldots$ \\
\hline Sniper & $85.2 \mathrm{gh}$ & $3.7 \mathrm{t}-\mathrm{v}$ & $0 \mathrm{f}$ & $65.1 \mathrm{e}-\mathrm{g}$ & $0.8 \mathrm{k}$ & $0 \mathrm{~d}$ \\
\hline Snowbird & $100.0 \mathrm{a}$ & $15.5 \mathrm{q}-\mathrm{t}$ & $0.8 \mathrm{f}$ & $\ldots$ & $\ldots$ & $\ldots$ \\
\hline Spector & $98.7 \mathrm{a}-\mathrm{c}$ & $\ldots$ & $\ldots$ & $95.0 \mathrm{ab}$ & $\ldots$ & $\ldots$ \\
\hline Spring Pac & $\ldots$ & $\ldots$ & $0 \mathrm{f}$ & $\ldots$ & $\ldots$ & $0.2 \mathrm{~d}$ \\
\hline Sun Blaster & $\ldots$ & $\ldots$ & $\ldots$ & $78.0 \mathrm{~d}$ & $\ldots$ & $\ldots$ \\
\hline Sun Devil & $93.0 \mathrm{a}-\mathrm{f}$ & $\ldots$ & $\ldots$ & $93.0 \mathrm{ab}$ & $\ldots$ & $\ldots$ \\
\hline Supercoach & $\ldots$ & $40.5 \mathrm{k}-\mathrm{o}$ & $\ldots$ & $\ldots$ & $37.2 \mathrm{~b}$ & $\ldots$ \\
\hline Synergene 352 & $\ldots$ & $40.0 \mathrm{k}-\mathrm{o}$ & $\ldots$ & $\ldots$ & $3.0 \mathrm{~h}-\mathrm{k}$ & $\ldots$ \\
\hline Target & $\ldots$ & $\ldots$ & $0.1 \mathrm{f}$ & $\ldots$ & $\ldots$ & $\ldots$ \\
\hline Telluride & $\ldots$ & $\ldots$ & $0 \mathrm{f}$ & $\ldots$ & $\ldots$ & $0 \mathrm{~d}$ \\
\hline Toronto & $\ldots$ & $\ldots$ & $0 \mathrm{f}$ & $\ldots$ & $\ldots$ & $\ldots$ \\
\hline Tradition & $100.0 \mathrm{a}$ & $20.1 \mathrm{pq}$ & 1.8 ef & $100.0 \mathrm{a}$ & $\ldots$ & $\ldots$ \\
\hline Tres Equis & $97.5 \mathrm{a}-\mathrm{c}$ & $\ldots$ & $\ldots$ & $100.0 \mathrm{a}$ & $\ldots$ & $\ldots$ \\
\hline Valley Green & $100.0 \mathrm{a}$ & $\ldots$ & $\ldots$ & $99.3 \mathrm{a}$ & $\ldots$ & $\ldots$ \\
\hline Valley Queen & $100.0 \mathrm{a}$ & $42.8 \mathrm{j}-\mathrm{m}$ & $\ldots$ & $100.0 \mathrm{a}$ & $7.8 \mathrm{f}-\mathrm{h}$ & $\ldots$ \\
\hline Vanguard 75 & $\ldots$ & $49.2 \mathrm{~g}-\mathrm{k}$ & $\ldots$ & $\ldots$ & $\ldots$ & $\ldots$ \\
\hline Van Max & $\ldots$ & $47.8 \mathrm{~g}-\mathrm{k}$ & $10.2 \mathrm{~b}$ & $\ldots$ & $7.4 \mathrm{f}-\mathrm{i}$ & $2.1 \mathrm{~b}$ \\
\hline Van Mor & $\ldots$ & $54.2 \mathrm{e}-\mathrm{j}$ & $\ldots$ & $\ldots$ & $\ldots$ & $\ldots$ \\
\hline Wellton & $100.0 \mathrm{a}$ & $\ldots$ & $\ldots$ & $100.0 \mathrm{a}$ & $\ldots$ & $\ldots$ \\
\hline Westland & $\ldots$ & $51.8 \mathrm{f}-\mathrm{k}$ & $\ldots$ & $\ldots$ & $8.3 \mathrm{fg}$ & $\ldots$ \\
\hline Wintergold & $\ldots$ & 29.8 n-p & $\ldots$ & $\ldots$ & $2.2 \mathrm{i}-\mathrm{k}$ & $\ldots$ \\
\hline Winterhaven & $\ldots$ & $59.8 \mathrm{~d}-\mathrm{g}$ & $\ldots$ & $\ldots$ & $40.4 \mathrm{ab}$ & $\ldots$ \\
\hline Winterking & $\ldots$ & $45.8 \mathrm{i}-\mathrm{k}$ & $\ldots$ & $\ldots$ & $2.3 \mathrm{i}-\mathrm{k}$ & $\ldots$ \\
\hline Wolverine & $\ldots$ & $4.4 \mathrm{~s}-\mathrm{V}$ & $\ldots$ & $99.0 \mathrm{ab}$ & $2.8 \mathrm{~h}-\mathrm{k}$ & $0 \mathrm{~d}$ \\
\hline Yuma & $\ldots$ & $46.2 \mathrm{i}-\mathrm{k}$ & $\ldots$ & $100.0 \mathrm{a}$ & $6.5 \mathrm{f}-\mathrm{j}$ & $\ldots$ \\
\hline \multicolumn{7}{|l|}{ Romaine } \\
\hline Apollo & $\ldots$ & $\ldots$ & $\ldots$ & $93.6 \mathrm{ab}$ & $0 \mathrm{k}$ & $0.2 \mathrm{~d}$ \\
\hline Big Green & $\ldots$ & $\ldots$ & $0 \mathrm{f}$ & $\ldots$ & $0 \mathrm{k}$ & $0.2 \mathrm{~d}$ \\
\hline BOS 9021 & $9.6 \mathrm{t}-\mathrm{w}$ & $\ldots$ & $\ldots$ & $8.8 \mathrm{n}$ & $\ldots$ & $\ldots$ \\
\hline Braveheart & $\ldots$ & $\ldots$ & $\ldots$ & $82.3 \mathrm{~cd}$ & $\ldots$ & $\ldots$ \\
\hline Clemente & $14.0 \mathrm{~s}-\mathrm{u}$ & $0.8 \mathrm{v}$ & $\ldots$ & $48.2 \mathrm{jk}$ & $0 \mathrm{k}$ & $0.1 \mathrm{~d}$ \\
\hline Coastal Star & $32.0 \mathrm{~m}$ & $2.3 \mathrm{uv}$ & $\ldots$ & $89.2 \mathrm{bc}$ & $\ldots$ & $0.2 \mathrm{~d}$ \\
\hline Conquistador & $12.7 \mathrm{t}-\mathrm{v}$ & $0 \mathrm{v}$ & $\ldots$ & $100.0 \mathrm{a}$ & $0 \mathrm{k}$ & $0.1 \mathrm{~d}$ \\
\hline Costa Rica \# 4 & $\ldots$ & $\ldots$ & $0 \mathrm{f}$ & $\ldots$ & $\ldots$ & $\ldots$ \\
\hline Darkland & $20.0 \mathrm{p}-\mathrm{s}$ & $0.7 \mathrm{v}$ & $\ldots$ & $50.7 \mathrm{i}-\mathrm{k}$ & $\ldots$ & $\ldots$ \\
\hline DF 7 & $12.9 \mathrm{t}-\mathrm{V}$ & $0.2 \mathrm{v}$ & $\ldots$ & $56.4 \mathrm{~h}-\mathrm{j}$ & $\ldots$ & $0.3 \mathrm{~d}$ \\
\hline Fresheart & $18.8 \mathrm{q}-\mathrm{t}$ & $\ldots$ & $\ldots$ & $7.6 \mathrm{n}$ & $4.6 \mathrm{f}-\mathrm{k}$ & $\ldots$ \\
\hline Green Forest & $26.3 \mathrm{~m}-\mathrm{p}$ & $\ldots$ & $\ldots$ & $77.9 \mathrm{~d}$ & $\ldots$ & $\ldots$ \\
\hline Green Towers & $19.2 \mathrm{q}-\mathrm{t}$ & $\ldots$ & $\ldots$ & 39.31 & $0 \mathrm{k}$ & $0 \mathrm{~d}$ \\
\hline King Louie & $6.9 \mathrm{vw}$ & $0.9 \mathrm{v}$ & $\ldots$ & $3.0 \mathrm{n}$ & $\ldots$ & $0.3 \mathrm{~d}$ \\
\hline Paragon PIC & $24.2 \mathrm{n}-\mathrm{q}$ & $1.2 \mathrm{v}$ & $\ldots$ & $66.6 \mathrm{eg}$ & $0 \mathrm{k}$ & $\ldots$ \\
\hline $\mathrm{PIC}$ & $29.2 \mathrm{mn}$ & $\ldots$ & $\ldots$ & $\ldots$ & $\ldots$ & $\ldots$ \\
\hline Robusto & $21.5 \mathrm{o}-\mathrm{q}$ & $0.9 \mathrm{v}$ & $\ldots$ & $45.4 \mathrm{kl}$ & $\ldots$ & $\ldots$ \\
\hline Slugger & $5.2 \mathrm{w}$ & $0.4 \mathrm{v}$ & $0 \mathrm{f}$ & $0.8 \mathrm{n}$ & $0 \mathrm{k}$ & $2.0 \mathrm{~b}$ \\
\hline Sunbelt & $\ldots$ & $0.4 \mathrm{v}$ & $0 \mathrm{f}$ & $\ldots$ & $0.8 \mathrm{k}$ & $2.2 \mathrm{~b}$ \\
\hline Triton & $16.7 \mathrm{r}-\mathrm{t}$ & $\ldots$ & $\ldots$ & $33.6 \mathrm{kl}$ & $\ldots$ & $0.2 \mathrm{~d}$ \\
\hline \multicolumn{7}{|l|}{ Green leaf } \\
\hline Big Star & $\ldots$ & $0.8 \mathrm{v}$ & $\ldots$ & $\ldots$ & $0.1 \mathrm{k}$ & $\ldots$ \\
\hline Marin & 89.7 d-g & $0.8 \mathrm{v}$ & $\ldots$ & $99.1 \mathrm{ab}$ & $0 \mathrm{k}$ & $\ldots$ \\
\hline North Star & $27.0 \mathrm{~m}-\mathrm{o}$ & $1.9 \mathrm{uv}$ & $\ldots$ & $29.0 \mathrm{~m}$ & $0.7 \mathrm{k}$ & $0.2 \mathrm{~d}$ \\
\hline Shining Star & $\ldots$ & $\ldots$ & $\ldots$ & $99.0 \mathrm{ab}$ & $\ldots$ & $\ldots$ \\
\hline Superstar & $\ldots$ & $\ldots$ & $\ldots$ & $\ldots$ & $2.5 \mathrm{i}-\mathrm{k}$ & $\ldots$ \\
\hline Two Star & $88.7 \mathrm{e}-\mathrm{g}$ & $2.9 \mathrm{uv}$ & $0 \mathrm{f}$ & $99.4 \mathrm{a}$ & $0 \mathrm{k}$ & $0.2 \mathrm{~d}$ \\
\hline \multicolumn{7}{|l|}{ Red leaf } \\
\hline Red Baron & $\ldots$ & $\ldots$ & $\ldots$ & $\ldots$ & $2.1 \mathrm{i}-\mathrm{k}$ & $\ldots$ \\
\hline Red Fox & $50.0 \mathrm{k}$ & $1.6 \mathrm{uv}$ & $\ldots$ & $\ldots$ & $0 \mathrm{k}$ & $\ldots$ \\
\hline Red Tide & $100.0 \mathrm{a}$ & $88.2 \mathrm{a}$ & $10.2 \mathrm{~b}$ & $100.0 \mathrm{a}$ & $2.2 \mathrm{i}-\mathrm{k}$ & $0.1 \mathrm{~d}$ \\
\hline Vulcan & $20.2 \mathrm{p}-\mathrm{s}$ & $2.8 \mathrm{uv}$ & $\ldots$ & $\ldots$ & $\ldots$ & $\ldots$ \\
\hline Western Red & $39.2 \mathrm{k}$ & $\ldots$ & $\ldots$ & $\ldots$ & $\ldots$ & $\ldots$ \\
\hline \multicolumn{7}{|l|}{ Butterhead } \\
\hline Connick & $\ldots$ & $66.5 \mathrm{~cd}$ & $0.3 \mathrm{f}$ & $\ldots$ & $\ldots$ & $\ldots$ \\
\hline Encanto & $65.7 \mathrm{j}$ & $\ldots$ & $\ldots$ & $\ldots$ & $\ldots$ & $\ldots$ \\
\hline Odyssey & $\ldots$ & $\ldots$ & $\ldots$ & $100.0 \mathrm{a}$ & $2.6 \mathrm{~h}-\mathrm{k}$ & $\ldots$ \\
\hline Optima & $86.2 \mathrm{f}-\mathrm{h}$ & $\ldots$ & $\ldots$ & $100.0 \mathrm{a}$ & $\ldots$ & $\ldots$ \\
\hline
\end{tabular}

plantings, disease incidence was recorded 1 and 21 October and 6 November 2003 for the 3 September planting; 22 November 2003 and 5 and 26 January 2004 for the 21 October planting; and 2 and 20 February and 22 March 2004 for the 18 December planting.

Analysis of data. CoStat statistical software (CoHort Software, Monterey, CA) was used to perform analysis of variance with the GLM procedure, to determine significant differences among main effects and any interactions, and to conduct linear regression analysis. Means were separated by LSD or Duncan's multiple range test.

\section{RESULTS}

The final incidence of Fusarium wilt recorded for each tested lettuce cultivar at crop maturity within each of the three planting dates in 2002 and 2003 is presented in Table 1. Within each planting date, significant differences in disease incidence were noted among lettuce cultivars. Seven and 16 lettuce cultivars in 2002 and 2003, respectively, were planted at each of the three planting dates for that year. In both years, the incidence of Fusarium wilt was significantly higher in the September planting compared with the October and December plantings (Table 2). In 2002, disease incidence also was significantly higher in the October planting compared with that in December. The mean soil temperatures at a depth of $10 \mathrm{~cm}$ during the September, October, and December plantings for 2002 and 2003 were 24 and $28^{\circ} \mathrm{C}, 15$ and $14^{\circ} \mathrm{C}$, and $14^{\circ} \mathrm{C}$, respectively (Table 2).

Initial symptoms of Fusarium wilt in September plantings began to appear on some plants approximately 14 days after seeding, when plants were thinned to a stand with $30 \mathrm{~cm}$ between seedlings in a row. Within each September planting, the mean incidence of Fusarium wilt increased significantly from the first to the second rating, and from the second to the third and final rating (Table 3). In all plantings, the level of Fusarium wilt increased as plant development progressed from juvenility to maturity and was significantly higher at the third rating at crop maturity compared with earlier ratings of younger immature plants. The stage of plant development at the time of each disease assessment in 2002 and 2003, expressed as the percentage of time elapsed from seeding to plant maturity, ranged from 33 to $48 \%, 60$ to $77 \%$, and $100 \%$ at the first, second, and third rating dates, respectively. For both years, the mean time from seeding to crop maturity during the September, October, and December plantings was 63, 91, and 101 days, respectively (Table 4). The mean soil temperature from seeding to first disease rating, from first to second rating, and from second to third and final rating, for the September, October, and December 
plantings, ranged from 28 to $21^{\circ} \mathrm{C}, 20$ to 2002-2003 season and from 32 to $22^{\circ} \mathrm{C}$, 19 to $12^{\circ} \mathrm{C}$, and 12 to $18^{\circ} \mathrm{C}$, respectively, in the 2003-2004 season (Table 4).

Four crisphead lettuce cultivars, Buccaneer, Desert Heat, Sharpshooter, and Sniper, were present in all three plantings in 2002 and in 2003. Among these cultivars, the main effects of time of planting and stage of plant development, as well as the interaction of time of planting $\times$ stage of plant development, stage of plant development $\times$ year, and time of planting $\times$ stage of plant development $\times$ year on the incidence of Fusarium wilt were significant (Table 5). On the other hand, the main effect of year and the interaction of time of planting $\times$ year were not significant. There $10^{\circ} \mathrm{C}$, and 12 to $18^{\circ} \mathrm{C}$, respectively, in the

also was a significant linear correlation $(r$ $=0.886, P<0.001)$, described by the equation $y=5.8 x-77$, between the final incidence of Fusarium wilt at plant maturity and the mean soil temperature at a depth of $10 \mathrm{~cm}$ (from seeding to plant maturity) for the lettuce cultivars Buccaneer, Desert Heat, Sharpshooter, and Sniper.

Among all types of lettuce in September plantings, only one and two cultivars of romaine in 2002 and 2003, respectively, reached maturity with $\leq 5 \%$ incidence of Fusarium wilt (Table 1), whereas the lowest incidence on crisphead, green leaf, red leaf, or butterhead cultivars was 73.7, 27.0, 20.2 , and $65.7 \%$, respectively, in 2002 and $62.1,29.0,100$, and $100 \%$, respectively, in 2003 (Tables 1 and 6). In October plant-

Table 2. Incidence of Fusarium wilt at crop maturity for lettuce cultivars grown in all three plantings in 2002 or 2003 in a field naturally infested with Fusarium oxysporum f. sp. lactucae

\begin{tabular}{lccccc}
\hline & \multicolumn{2}{c}{ Plants dead or diseased $(\boldsymbol{\%})^{\mathbf{x}}$} & & \multicolumn{2}{c}{ Mean soil temp $\left({ }^{\mathbf{C}} \mathbf{C}\right)^{\mathbf{y}}$} \\
\cline { 2 - 3 } \cline { 5 - 6 } Planting time $^{\mathbf{z}}$ & $\mathbf{2 0 0 2}$ & $\mathbf{2 0 0 3}$ & & $\mathbf{2 0 0 2}$ & $\mathbf{2 0 0 3}$ \\
\hline September & $92.3 \mathrm{a}$ & $74.2 \mathrm{a}$ & & 24 & 28 \\
October & $15.1 \mathrm{~b}$ & $5.1 \mathrm{~b}$ & & 15 & 14 \\
December & $2.0 \mathrm{c}$ & $0.7 \mathrm{~b}$ & & 14 & 14 \\
\hline
\end{tabular}

${ }^{\mathrm{x}}$ Each plant within a plot was determined to be diseased if the plant was dead or stunted and displayed the typical wilting and yellowing associated with Fusarium wilt of lettuce. Each value for 2002 and 2003 was derived from seven and 16 lettuce cultivars, respectively. Values in each column followed by a different letter are significantly different according to the LSD test.

${ }^{\mathrm{y}}$ Mean soil temperature at a depth of $10 \mathrm{~cm}$ from seeding to plant maturity.

${ }^{\text {z }}$ Planting dates were 7 Sep, 17 Oct, and 6 Dec in 2002 and 3 Sep, 21 Oct, and 18 Dec in 2003. Final disease incidence at crop maturity was determined for each respective planting in 2002 on 8 Nov 2002, 11 Jan and 22 Mar 2003, and for 2003 plantings on 6 Nov 2003, 26 Jan and 22 Mar 2004.

Table 3. Incidence of Fusarium wilt at the first, second, and final disease rating date within each lettuce planting grown in a field naturally infested with Fusarium oxysporum f. sp. lactucae

\begin{tabular}{|c|c|c|c|c|c|c|}
\hline \multirow{3}{*}{$\begin{array}{l}\text { Disease } \\
\text { rating }^{z}\end{array}$} & \multicolumn{6}{|c|}{ Plants dead or diseased $(\%)^{y}$} \\
\hline & \multicolumn{3}{|c|}{ Planting date: 2002-2003 } & \multicolumn{3}{|c|}{ Planting date: 2003-2004 } \\
\hline & $7 \mathrm{Sep}$ & 17 Oct & 6 Dec & 3 Sep & 21 Oct & 18 Dec \\
\hline First & $15.3 \mathrm{c}$ & $1.3 \mathrm{c}$ & $0 \mathrm{~b}$ & $44.2 \mathrm{c}$ & $0.7 \mathrm{~b}$ & $0 \mathrm{~b}$ \\
\hline Second & $75.0 \mathrm{~b}$ & $12.8 \mathrm{~b}$ & $0.2 \mathrm{~b}$ & $64.0 \mathrm{~b}$ & $2.4 \mathrm{~b}$ & $0.1 \mathrm{~b}$ \\
\hline Last & $96.8 \mathrm{a}$ & $37.4 \mathrm{a}$ & $1.8 \mathrm{a}$ & $80.1 \mathrm{a}$ & $7.1 \mathrm{a}$ & $0.6 \mathrm{a}$ \\
\hline
\end{tabular}

${ }^{\mathrm{y}}$ Each plant within a plot was determined to be diseased if the plant was dead or stunted and displayed the typical wilting and yellowing associated with Fusarium wilt of lettuce. Each value for the September, October, and December plantings in 2002 was derived from 38, 34, and 40 cultivars, respectively, and in 2003 from 60, 48, and 41 cultivars, respectively. Values in each column followed by a different letter are significantly different according to the LSD test.

${ }^{\mathrm{z}}$ The first, second, and last determinations of Fusarium wilt incidence for 2002 plantings in September, October, and December were conducted 25, 37, and 62 days; 37, 58, and 86 days; and 50, 71, and 107 days after seeding, respectively. For 2003 plantings in the same months, disease was evaluated 28, 48, and 64 days; 32, 76, and 97 days; and 46, 64, and 95 days after seeding, respectively. ings, all romaine cultivars had $\leq 5 \%$ incidence of Fusarium wilt at maturity, whereas disease incidence among the 37 and 30 tested cultivars of crisphead lettuce in 2002 and 2003 ranged from 0.8 to $66.8 \%$ and 0.3 to $43.3 \%$, respectively. For December plantings, 82 and $88 \%$ of tested cultivars, including all romaine entries, reached maturity with $\leq 1 \%$ incidence of Fusarium wilt (Tables 1 and 6).

\section{DISCUSSION}

Planting date had a profound effect on the final incidence of Fusarium wilt in these studies. One prominent difference among planting dates was soil temperature. In the September plantings of 2002 and 2003, the mean incidence of Fusarium wilt was $88 \%$ and the mean soil temperature was $26^{\circ} \mathrm{C}$. Disease incidence for both October plantings was $22 \%$, and the mean soil temperature was $14^{\circ} \mathrm{C}$, and for both December plantings, mean disease incidence was $1 \%$ and the mean soil temperature also was $14^{\circ} \mathrm{C}$. Lower disease incidence in December compared with October plantings, when mean soil temperatures were the same, may be explained by the change in soil temperatures as the October and December lettuce plantings grew and reached maturity. In the October plantings, the recorded mean soil temperature for 2002 and 2003 from seeding to first disease rating, first rating to second rating, and second rating to third (final) rating was 20,12 , and $11^{\circ} \mathrm{C}$, respectively; whereas during December plantings, mean recorded soil temperatures at these respective periods of time were 12,14 , and $18^{\circ} \mathrm{C}$. Higher soil temperatures early in the development of the lettuce plant, as recorded in October plantings, apparently were more favorable for disease development than higher soil temperature near the end of the crop development cycle, as recorded in December plantings. Hubbard and Gerik (10) reported that an isolate of the lettuce Fusarium wilt pathogen grew in culture from 8 to $32^{\circ} \mathrm{C}$, with maximum growth at $28^{\circ} \mathrm{C}$. In our studies, a greater incidence of Fusarium wilt was recorded among lettuce plantings when mean soil temperatures were 24 and $28^{\circ} \mathrm{C}$ compared with disease levels at 14 and $15^{\circ} \mathrm{C}$. Temperatures ranging from 25 to $35^{\circ} \mathrm{C}$, compared with 10 to

Table 4. Mean soil temperature during intervals of time before Fusarium wilt incidence ratings

\begin{tabular}{|c|c|c|c|c|c|c|c|c|}
\hline \multirow[b]{2}{*}{ Planting date } & \multicolumn{2}{|c|}{ Seeding to first rating } & \multicolumn{2}{|c|}{ First rating to second rating } & \multicolumn{2}{|c|}{ Second rating to final rating } & \multicolumn{2}{|c|}{ Seeding to final rating } \\
\hline & Time $^{y}$ (days) & Temp. ${ }^{\mathrm{z}}\left({ }^{\circ} \mathbf{C}\right)$ & Time (days) & Temp. $\left({ }^{\circ} \mathbf{C}\right)$ & Time (days) & Temp. $\left({ }^{\circ} \mathbf{C}\right)$ & Time (days) & Temp. $\left({ }^{\circ} \mathrm{C}\right)$ \\
\hline \multicolumn{9}{|l|}{ 2002-03 } \\
\hline $7 \mathrm{Sep}$ & 25 & 28 & 12 & 24 & 25 & 21 & 62 & 24 \\
\hline $17 \mathrm{Oct}$ & 37 & 20 & 21 & 14 & 28 & 10 & 86 & 15 \\
\hline $6 \mathrm{Dec}$ & 50 & 12 & 21 & 15 & 36 & 18 & 107 & 14 \\
\hline \multicolumn{9}{|l|}{ 2003-04 } \\
\hline $3 \mathrm{Sep}$ & 28 & 32 & 20 & 28 & 16 & 32 & 64 & 28 \\
\hline $21 \mathrm{Oct}$ & 32 & 19 & 44 & 11 & 21 & 12 & 97 & 14 \\
\hline $18 \mathrm{Dec}$ & 46 & 12 & 18 & 12 & 31 & 18 & 95 & 14 \\
\hline
\end{tabular}

y Elapsed time in days.

${ }^{\mathrm{z}}$ Mean soil temperature at a depth of $10 \mathrm{~cm}$. 
$20^{\circ} \mathrm{C}$, are associated with higher levels of Fusarium wilt on several other crops as well. Punja et al. (17) noted that symptoms of Fusarium wilt on muskmelon, caused by F. oxysporum f. sp. melonis, occurred when temperatures exceeded the 25 to $30^{\circ} \mathrm{C}$ range. For Fusarium wilt on banana, caused by $F$. oxysporum f. sp. cubense, Peng et al. (16) reported an increase in disease severity as temperature increased from 24 to $34^{\circ} \mathrm{C}$, whereas at $14^{\circ} \mathrm{C}$, no wilt symptoms were evident even though the pathogen could be recovered from within banana plant tissue. Fusarium wilt of carnation, caused by F. oxysporum f. sp. dianthi, was most severe at 25 to $26^{\circ} \mathrm{C}$, whereas plants below $18^{\circ} \mathrm{C}$ remained symptomless (1). Symptoms of Fusarium wilt of chickpea were less severe from 10 to $20^{\circ} \mathrm{C}$ than from 25 to $30^{\circ} \mathrm{C}(2)$, and a constant temperature of $35^{\circ} \mathrm{C}$ was most favorable for disease development on chrysanthemum (6).

Initial symptoms of Fusarium wilt appeared as early as 14 days after seeding on some lettuce cultivars planted in September. The number of plants with symptoms continued to increase from this time to maturity, and disease incidence for all plantings was significantly higher at maturity compared with the first rating early in crop development. Evaluation of plant material for resistance to Fusarium wilt should be conducted at temperatures expected in the field and continued for the duration of time required for plants to mature to generate resistance evaluation data from greenhouse or growth chamber studies that will accurately predict performance in fields infested with $F$. oxysporum $\mathrm{f}$. sp. lactucae.

Fusarium wilt of lettuce is an emerging disease in the southwestern Arizona production region. The 27 fields currently known to contain $F$. oxysporum f. sp. lactucae, ranging in size from 4 to 16 ha, currently represent $<1 \%$ of the total land area devoted to lettuce production in this area. Disease incidence at the time of initial detection of Fusarium wilt ranged from fields with less than 10 symptomatic plants to the occasional field with virtually $100 \%$ infected plants. The number of fields infested with $F$. oxysporum f. sp. lactucae will likely increase as contaminated soil is inadvertently introduced into fields cur-

Table 5. Analysis of variance table evaluating the effects of time of planting, stage of plant development, year, and their interactions on the incidence of Fusarium wilt with four lettuce cultivars (Buccaneer, Desert Heat, Sharpshooter, and Sniper) grown in all three plantings in 2002 and 2003 in a field naturally infested with Fusarium oxysporum f. sp. lactucae

\begin{tabular}{lccc}
\hline & & \multicolumn{2}{c}{ Incidence of Fusarium wilt } \\
\cline { 3 - 4 } Source $^{\mathbf{x}}$ & df $^{\mathbf{y}}$ & $\boldsymbol{F}$ & $\boldsymbol{P}>\boldsymbol{F}^{\mathbf{z}}$ \\
\hline Time of planting (TP) & 2 & 358.1 & $<0.0001^{* * *}$ \\
Stage of plant development (PD) & 2 & 54.0 & $<0.0001^{* * *}$ \\
Year (Y) & 1 & 0.9 & $0.3376 \mathrm{~ns}$ \\
TP $\times$ PD & 4 & 35.2 & $<0.0001^{* * *}$ \\
TP $\times$ Y & 2 & 1.7 & $0.1821 \mathrm{~ns}$ \\
PD $\times$ Y & 2 & 10.8 & $<0.0001^{* * *}$ \\
TP $\times$ PM $\times$ Y & 4 & 4.8 & $0.0011^{* *}$ \\
\hline
\end{tabular}

${ }^{x}$ Sources of variation. Time of planting: September, October, and December. Stage of plant development, expressed as the percentage of time elapsed from seeding to plant maturity at each of the three disease rating dates for each planting in 2002 and 2003, ranged from 33 to $48 \%$ at the first disease rating date, 60 to $77 \%$ at the second rating date, and 100\% for the third rating date. Years: 2002 and 2003 .

${ }^{y}$ Degrees of freedom.

$\mathrm{z} * *, * * *=$ significant at $P<0.01$ and $P<0.001$, respectively.

rently free of the pathogen through farming activities such as laser-leveling, disking, and other seed-bed preparation activities, movement of mud-encrusted irrigation pipe used to germinate seed, cultivation, and harvesting crew operations. Growers and harvesters are strongly encouraged to minimize dissemination of the Fusarium wilt pathogen. All agricultural land in southwestern Arizona is double-cropped, i.e., two crops per year, which increases the potential for dispersion of the pathogen. After lettuce or another winter vegetable has been harvested, spring and summer crops, such as melons, cotton, wheat, corn, safflower, or Sudan grass, are planted and grown. Sanitation practices directed at a lettuce pathogen may easily be neglected during the production of these crops.

Seed transmission of $F$. oxysporum f. sp. lactucae is another means of pathogen dissemination that may account for the appearance of Fusarium wilt on lettuce in geographically distant areas, such as California, Taiwan, Iran, and Italy, at least 35 years after initial discovery in Japan. Garibaldi et al. (7) found that nine of 27 samples of lettuce seed obtained from commercial seed lots planted in fields that were subsequently affected by Fusarium wilt in Italy were contaminated by $F$. $o x$ ysporum. Three of the isolates were pathogenic to lettuce and exhibited a level of pathogenicity similar to that of isolates of F. oxysporum f. sp. lactucae originally isolated from infected plants in Italy, the United States, and Taiwan.

The primary management tool for Fusarium wilt on other crops is the use of resistant cultivars. Our evaluation of crisphead lettuce cultivars, the primary type of lettuce grown in southwestern Arizona, revealed no resistant or tolerant cultivar available for early-season planting in September. Long-term management of Fusarium wilt, using current planting schedules and farming practices, will require development of resistant crisphead and other

Table 6. Incidence of Fusarium wilt at crop maturity among types of lettuce planted in September, October, and December in 2002 or 2003 in a field naturally infested with Fusarium oxysporum f. sp. lactucae ${ }^{z}$

\begin{tabular}{|c|c|c|c|c|c|c|c|c|c|}
\hline \multirow[b]{3}{*}{ Lettuce type } & \multicolumn{3}{|c|}{ September planting } & \multicolumn{3}{|c|}{ October planting } & \multicolumn{3}{|c|}{ December planting } \\
\hline & \multirow{2}{*}{$\begin{array}{c}\text { Cultivars } \\
\text { tested }\end{array}$} & \multicolumn{2}{|c|}{ Disease incidence (\%) } & \multirow{2}{*}{$\begin{array}{c}\text { Cultivars } \\
\text { tested }\end{array}$} & \multicolumn{2}{|c|}{ Disease incidence $(\%)$} & \multirow{2}{*}{$\begin{array}{c}\text { Cultivars } \\
\text { tested }\end{array}$} & \multicolumn{2}{|c|}{ Disease incidence $(\%)$} \\
\hline & & Range & Mean & & Range & Mean & & Range & Mean \\
\hline \multicolumn{10}{|l|}{$2002-2003$} \\
\hline Crisphead & 38 & $73.7-100$ & 96.9 & 37 & $0.8-66.8$ & 35.1 & 33 & $0-17.8$ & 1.9 \\
\hline Romaine & 15 & $5.2-32.0$ & 17.9 & 10 & $0-2.3$ & 0.8 & 4 & 0 & 0 \\
\hline Green leaf & 3 & $27.0-89.7$ & 68.5 & 4 & $0.8-2.9$ & 1.6 & 1 & 0 & 0 \\
\hline Red leaf & 4 & $20.2-100$ & 52.4 & 3 & $1.6-88.2$ & 30.9 & 1 & 10.2 & 10.2 \\
\hline Butterhead & 2 & $65.7-86.2$ & 76.0 & 1 & 66.5 & 66.5 & 1 & 0.3 & 0.3 \\
\hline \multicolumn{10}{|l|}{ 2003-2004 } \\
\hline Crisphead & 37 & $62.1-100$ & 91.2 & 30 & $0.3-43.3$ & 10.9 & 27 & $0-5.6$ & 0.7 \\
\hline Romaine & 16 & $0.8-100$ & 50.2 & 9 & $0-4.6$ & 0.6 & 11 & $0-2.2$ & 0.5 \\
\hline Green leaf & 4 & $29.0-99.4$ & 81.6 & 5 & $0-2.5$ & 0.7 & 2 & 0.2 & 0.2 \\
\hline Red leaf & 1 & 100 & 100 & 3 & $0-2.2$ & 1.4 & 1 & 0.1 & 0.1 \\
\hline Butterhead & 2 & 100 & 100 & 1 & 2.6 & 2.6 & 0 & $\ldots$ & $\ldots$ \\
\hline
\end{tabular}

${ }^{\mathrm{z}}$ Each plant within a plot was determined to be diseased if the plant was dead or stunted and displayed the typical wilting and yellowing associated with Fusarium wilt of lettuce. 
lettuce types that can be grown successfully in infested fields when temperatures favor disease development. On the other hand, for the short-term, some important disease management strategies can be implemented. The long planting and growing season, starting with first sowing of seed in early September and ending with final harvest in late March, coupled with the effect of temperature on disease development, allows growers to plant some crisphead cultivars in the cool soil during December in the presence of $F$. oxysporum f. sp. lactucae and expect minimal to no crop loss. Furthermore, several romaine lettuce cultivars could be successfully planted from October through December in fields infested with the pathogen. Other vegetable crops produced in the area, primarily broccoli, cauliflower, and cabbage, can also be grown on land known to be infested with $F$. $o x$ ysporum f. sp. lactucae. These short-term management practices should remain effective until the pathogen has spread to such a large proportion of available land that timing and rotation strategies will no longer be usable. Hopefully, by that time some lettuce cultivars with a high level of tolerance or resistance to the Fusarium wilt pathogen will be available.

Management strategies directed at lowering inoculum levels of $F$. oxysporum $\mathrm{f}$. sp. lactucae in infested fields could be beneficial in reducing spread of the pathogen both within and between fields. Soil solarization, in particular, may be a useful technique to reduce population levels of the pathogen and disease incidence in infested fields. Chellemi et al. (3) found no difference in the incidence of Fusarium wilt of tomato in plots solarized 40 to 55 days compared with plots fumigated with a mixture of methyl bromide + chloropicrin. Lodha (11) noted that soil solarization for 15 days led to significant reduction in the incidence of Fusarium wilt of cumin. Experiments are in progress to assess the potential of soil solarization as a management tool for Fusarium wilt of lettuce in southwestern Arizona production fields.

\section{LITERATURE CITED}

1. Ben-Yephet, Y., and Shtienberg, D. 1994. Effects of solar radiation and temperature on Fusarium wilt in carnation. Phytopathology 84:1416-1421.

2. Bhatti, M. A., and Kraft, J. M. 1992. Effects of inoculum density and temperature on root rot and wilt of chickpea. Plant Dis. 76:50-54.

3. Chellemi, D. O., Olson, S. M., Mitchell, D. J., Secker, I., and McSorley, R. 1997. Adaptation of soil solarization to the integrated management of soilborne pests of tomato under humid conditions. Phytopathology 87:250-258.

4. Fujinaga, M., Ogiso, H., Tuchiya, N., Saito, H., Yamanaka, S., Nozue, M., and Kojima, M. 2003. Race 3, a new race of Fusarium oxysporum f.sp. lactucae determined by differential system with commercial cultivars. J. Gen. Plant Pathol. 69:23-28.

5. Fujinaga, M., Ogiso, H. T. N., and Saito, H. 2001. Physiological specialization of Fusarium oxysporum f.sp. lactucae, a causal organism of Fusarium root rot of crisp head lettuce in Japan. J. Gen. Plant Pathol. 67:205-206.

6. Gardiner, D. C., Horst, R. K., and Nelson, P. E. 1987. Symptom enhancement of Fusarium wilt of chrysanthemum by high temperatures. Plant Dis. 71:1106-1109.
7. Garibaldi, A., Gilardi, G., and Gullino, M. L. 2004. Seed transmission of Fusarium oxysporum f.sp. lactucae. Phytoparasitica 32:6165.

8. Garibaldi, A., Gilardi, G., and Gullino, M. L. 2002. First report of Fusarium oxysporum on lettuce in Europe. Plant Dis. 86:1052.

9. Huang, J. H., and Lo, C. T. 1998. Wilt of lettuce caused by Fusarium oxysporum in Taiwan. Plant Pathol. Bull. 7:150-153.

10. Hubbard, J. C., and Gerik, J. S. 1993. A new wilt disease of lettuce incited by Fusarium oxysporum f. sp. lactucum forma specialis nov. Plant Dis. 77:750-754.

11. Lodha, S. 1995. Soil solarization, summer irrigation and amendments for the control of Fusarium oxysporum f.sp. cumini and Macrophomina phaseolina in arid soils. Crop Prot. 14:215-219.

12. Matheron, M. E., and Koike, S. T. 2003. First report of Fusarium wilt of lettuce caused by Fusarium oxysporum f. sp. lactucae in Arizona. Plant Dis. 87:1265

13. Matuo, T., and Motohashi, S. 1967. On Fusarium oxysporum $\mathrm{f}$. sp. lactucae $\mathrm{n}$. f. causing root rot of lettuce. Trans. Mycol. Soc. Jpn. 32:13-15.

14. McCreight, J. D., Matheron, M., Tickes, B., and Platts, B. Fusarium wilt race 1 on lettuce. HortScience In press.

15. Millani, M. J., Etebarian, H. R., and Alizadeh, A. 1999. Occurrence of Fusarium wilt of lettuce in Shahre-Ray, Varamin and Karaj areas. Iran. J. Plant Pathol. 35:44-45.

16. Peng, H. X., Sivasithamparam, K., and Turner D. W. 1999. Chlamydospore germination and Fusarium wilt of banana plantlets in suppressive and conducive soils are affected by physical and chemical factors. Soil Biol. Biochem. 31:1363-1374.

17. Punja, Z. K., Parker, M., and Elmhirst, J. F 2001. Fusarium wilt of field-grown muskmelon in British Columbia. Can. J. Plant Pathol. 23:403-410. 